\title{
Editorial coment to Visual prostate symptom score (VPSS). Is it an effective alternative for the assessment of lower urinary tract symptoms? Experience in a reference center in southeastern Mexico
}

\section{Comentario editorial a Visual Prostate Symptom Score (VPSS). ¿Es una alternativa eficaz para la evaluación de los síntomas del tracto urinario inferior? Experiencia en un centro de referencia en el sureste de México}

Paulina Bueno-García-Reyes. ${ }^{*}$

Corresponding author:

*Paulina Bueno-

García-Reyes. Vasco de Quiroga 15, Belisario Domínguez Secc 16, Tlalpan, 14080 Ciudad de México, México. Email: paulina_bgr@ hotmail.com
I want to congratulate the author of the study "Visual Prostate Symptom Score (VPSS): Is It An Effective Alternative For The Assessment Of Lower Urinary Tract Symptoms? Experience In A Reference Center In Southeastern Mexico" as it points out the importance of accessibility to a basic, yet important, tool to assess lower urinary tract symptoms (LUTS). By using a visual version of the widely known International Prostate Symptom Score (IPSS), it is possible to reach populations in which language and cultural level could be an obstacle, promoting best practice for urological patients in Mexico.

The importance of standardized questionnaires and instruments, such as the IPSS, has been repeatedly highlighted for evaluation, management, and follow-up of LUTS. They are included in the most relevant LUTS international guidelines. Furthermore, IPSS can provide objective comparison before and after treatment, it being conservative or invasive. Most of the literature describing different treatment options for LUTS and bladder outlet obstruction (BOO) use IPSS to measure the success of the intervention.

We know that the presence of LUTS is not diagnostic of BOO and in consequence IPSS is not enough on its own to adequately diagnose BOO since it is not disease specific.(1) But if used in addition to other questionnaires and diagnostic tools it can provide valuable information to guide treatment.

Citation: Bueno-García-Reyes P. Editorial coment to Visual prostate symptom score (VPSS). Is it an effective alternative for the assessment of lower urinary tract symptoms? Experience in a reference center in southeastern Mexico. Rev Mex Urol. 2021;81(6):pp. 1-2

${ }^{1}$ Secretaría de Salud, Instituto Nacional de Ciencias Médicas y Nutrición Salvador Zubirán Ciudad de México, México.

Received: December 7, 2021

Accepted: December 10, 2021

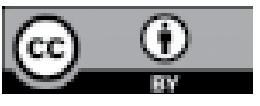




\section{Editorial coment to Visual prostate symptom score (VPSS).Is it an effective... Bueno-García-Reyes P., et al.}

The recently published exploratory findings from the UPSTREAM Trial by GJ Young et al,(2) suggest that IPSS $>16$, IPSS QoL $>8$, along with an ICIQ voiding subscale score $>8$, ICIQ score $>18$, Qmax $<9.8 \mathrm{ml} / \mathrm{s}$, and age $<74$ yr can predict success from surgery for LUTS. So, four out of the six recommended variables to predict a successful outcome from surgical treatment for LUTS, are standardized questionnaires (IPSS and ICIQ). If such non-invasive, cheap, risk free and easy to apply instruments (except for cultural or language barriers) are not used, we lose vital information that can lead to impaired decision making, wrong management and therefore poor outcomes.

As for other urological conditions, such as nocturia, incontinence, erectile dysfunction, overactive bladder, amongst others, we shall encourage the use of standardized questionnaires whenever possible and endeavor to make them accessible for patients with limitations.

We have a particularly challenging situation in countries like Mexico where education is not universal and with such a diversity of cultures and languages, but we still have the responsibility to provide a good standard of care.

\section{References}

1. D'Silva KA, Dahm P, Wong CL. Does this man with lower urinary tract symptoms have bladder outlet obstruction?: The Rational Clinical Examination: a systematic review. JAMA. 2014 Aug 6;312(5):535-42. doi: https://doi. org/10.1001/jama.2014.5555

2. Young GJ, Metcalfe C, Lane JA, Lewis AL, Abrams P, Blair PS, et al. Prostate Surgery for Men with Lower Urinary Tract Symptoms: Do We Need Urodynamics to Find the Right Candidates? Exploratory Findings from the UPSTREAM Trial. Eur Urol Focus. 2021;S2405-4569(21)00310-2. doi: https://doi. org/10.1016/j.euf.2021.11.010 